\title{
Observational Constraints on Cosmological Models
}

\author{
T. Padmanabhan \\ Inter-University Centre for Astronomy and Astrophysics, Post Bag ל, \\ Ganeshkhind, Pune - 411007 , India \\ email: paddy@iucaa.ernet.in
}

\begin{abstract}
Cosmological models for structure formation are severely constrained by several of the recent observational results. We now have observations which probe the power spectrum of fluctuations from about $0.5 h^{-1} \mathrm{Mpc}$ to $3000 h^{-1} \mathrm{Mpc}$. These probes and the constraints they imply on models for structure formation are reviewed.
\end{abstract}

\section{Recipe for the Universe}

Models for cosmological structure formation assume that small perturbations in the energy density, which originated at very early epochs, have grown via gravitational instability, leading to the structures we see today. In most of the models, these perturbations are generated by processes which are supposed to have taken place in the very early universe (say, at $z \gtrsim 10^{18}$ ) and has an initial power spectrum $P_{\text {in }}(k) \simeq A k$. Since each logarithmic interval in $k$ space will contribute to the energy density an amount $\triangle_{p}^{2}(k) \equiv d \sigma^{2} / d(\ln k)=\left(k^{3} P(k) / 2 \pi^{2}\right)$ we find that $\triangle_{p}^{2} \propto k^{4}$ if $P \propto k$. The contribution to the gravitational potential from the same range will be $\triangle_{\varphi}^{2} \propto \triangle_{\rho}^{2} / k^{4}$ which is independent of $k$ if $\triangle_{\rho}^{2} \propto k^{4}$. Such a "scale-invariant" spectrum is theoretically quite attractive and arises in several models including seeded models and inflationary models.

Given a slightly perturbed Friedmann model, with small inhomogeneities described by a power spectrum $P\left(k, z_{\text {in }}\right)$ at a high redshift $z=z_{\text {in }}$, we can predict unambigiously the power spectrum $P\left(k, z_{D}\right)$ at the epoch of decoupling, $z=z_{D} \approx 10^{3}$. This is possible because we can use linear perturbation theory during this epoch. The shape of the spectrum at $z=z_{D}$ will not be a pure power law since the gravitational amplification is wavelength-dependent. In general, the power at small scales is suppressed due to various physical processes. The exact shape at $z=z_{\mathrm{D}}$ depends on the kind of dark matter present in the universe. In a universe dominated by "hot dark matter" (HDM) particles of mass $m \simeq$ $30 \mathrm{eV}$, the power per logarithmic interval, $\triangle_{\rho}(k)$ is peaked at $k=k_{\max } \equiv 0.11$ $\mathrm{Mpc}^{-1}(\mathrm{~m} / 30 \mathrm{eV})$ and falls exponentially for $k>k_{\max }$. Hence, in these models, the scale $k=k_{\max }$ will go nonlinear first and smaller structures have to form by fragmentation. If the universe is dominated by "cold dark matter" (CDM) particles with mass $m \gtrsim 35 \mathrm{GeV}$, then $\Delta(k)$ is a gently increasing function of $k$ for small $k$. If we set $P(k) \propto k^{n}$ locally, the index $n$ changes from 1 at $k^{-1} \gtrsim 200 h^{-1} \mathrm{Mpc}$ to 0 at $k^{-1} \simeq 10 h^{-1} \mathrm{Mpc}$ and to about (-2) at $k^{-1} \simeq 1 h^{-1}$ 
Mpc. In such models small scales will go nonlinear first and the structure will develop hierarchically.

The situation is more complicated if two kinds of dark matter are present or if the cosmological constant is non-zero. The presence of the cosmological constant adds to the power at large scales but suppresses the growth of perturbations at small scales. A similar effect takes place if a small fraction of the dark matter is hot and the bulk of it is cold (e.g. $\Omega_{\mathrm{HDM}} \simeq 0.3, \Omega_{C D M} \simeq 0.7$ ). In both cases there will be more power at large scales and less power at small scales, compared to standard CDM model. The spectrum $\Delta(k)$ is still a gently increasing function of $k$ and small scales go nonlinear first.

The fact, that one can compute the power spectrum at $z \simeq z_{D}$ analytically, allows one to predict large scale anisotropies in the cosmic microwave background (CMBR) unambiguously in any given model. Comparing this prediction with the anisotropy observed by COBE one can fix the amplitude $A$ of the power spectrum. For a wide class (e.g. Padmanabhan \& Narasimha 1992) of the models, $\triangle(k) \cong 10^{-3}(k L)^{2}$ with $L \simeq(24 \pm 4) h^{-1} \mathrm{Mpc}$ for $k^{-1} \gtrsim 80 h^{-1} \mathrm{Mpc}$. For CDM-like models the function $\triangle(k)$ flattens out at larger $k$ and is about unity around $k^{-1} \simeq 8 h^{-1} \mathrm{Mpc}$. In pure HDM models, $\triangle(k)$ has a maximum value of $\triangle_{m} \simeq 0.42 h^{-2}(m / 30 e V)^{2}$ at $k_{m} \simeq 0.11 \mathrm{Mpc}^{-1}(m / 30 \mathrm{eV})$ and decreases exponentially at $k \gtrsim k_{m}$.

The evolution of the power spectrum after decoupling (for $z<z_{\mathrm{D}}$ ) is more difficult to work out theoretically. In general, the power spectrum grows in amplitude (preserving the shape), as long as the perturbations are small. In this case, we can write $\Delta(k, z)=\left[f(z) / f\left(z_{D}\right)\right] \Delta\left(k, z_{D}\right)$ for $z<z_{D}$. For example, in CDM models with $\Omega=1, f(z)=(1+z)^{-1}$; thus $\Delta(k)$ grows by a factor $10^{3}$ at all scales between the epoch of decoupling $\left(z_{D} \simeq 10^{3}\right)$ and the present epoch $(z=0)$, if we assume that linear theory is valid at all scales. The resulting $\triangle_{0}(k)$, obtained by linear extrapolation, is often used to specify the properties of the models. This spectrum correctly describes the power at large scales (say, $k^{-1} \gtrsim 30 h^{-1} \mathrm{Mpc}$ ) where $\Delta_{0} \lesssim 0.1$. Fig. 1 shows the density contrast $\sigma(R)$ computed from the linearly extrapolated power spectrum $\triangle_{0}(k)$ in four different models. The density contrast $\sigma(R)$ measures the rms fluctuations in mass within a randomly placed sphere of radius $R$; up to factors of order unity, $\sigma(R) \simeq \triangle(k \simeq$ $R^{-1}$ ).

At small scales, the true power $\Delta_{\text {true }}(k)$ will be larger than $\Delta_{0}(k)$ due to nonlinear effects. There have been several attempts in the literature to understand the form of $\triangle_{\text {true }}(k)$ at $k^{-1} \lesssim 30 h^{-1} \mathrm{Mpc}$. Since dark matter particles interact only through gravity, it is possible to study the formation of dark matter structures by numerical simulations. For a wide class of models, one can relate (Bagla \& Padmanabhan 1993; Hamilton et al. 1991) the nonlinear and linear density contrasts by a set of power laws of the form: $\sigma_{N L}^{2}(a, x)=A\left[\sigma_{L}^{2}(a, l)\right]^{n}$ with $l^{3}=x^{3}\left(1+\sigma_{N L}^{2}\right)$ where $A=n=1$ for $\sigma_{L}^{2} \leq 1.2 ; A=0.794, n=2.9$ for $1.2 \leq \sigma_{L}^{2} \leq 6.0$ and $A=9.12, n=1.55$ for $\sigma_{L}^{2} \geq 6.0$. This relation shows that $\sigma_{N L}$ is steeper than $\sigma_{L}$ at small scales.

The evolution at small scales is also complicated due to baryonic physics. Since baryons can dissipate energy and sink to the minima of the dark matter potential wells, the statistical properties of visible galaxies and dark matter halos could be quite different. One should also remember that, in hierarchical 
models, considerable amount of merging takes place at small scales. It is usual to quantify our ignorance at these scales by a 'bias' (acronym for 'Basic Ignorance of Astrophysical Scenarios') factor $b$ and write $\xi_{\text {gal }}(r)=b^{2} \xi_{\text {mass }}(r)$. Such a parametrisation is useful only if $b$ is idependent of scale and morphology of galaxies, which seems to be somewhat unlikely. Since small scale observations are based on galactic properties, while theoretical calculations usually deal with the underlying mass distribution, any scale (or morphology) dependence of $b$ could have important bearing on the comparison of observations with models.

\section{Probing the Power Spectrum}

One of the direct ways of constraining the models is to estimate the density contrast $\sigma_{\text {obs }}(R)$ from observations at different scales and compare it with the theoretically predicted values. Fortunately, we now have observational probes covering four decades of scales from $10^{-1} \mathrm{Mpc}$ to $10^{3} \mathrm{Mpc}$; these are summarised in Table 1.

The first column of the Table 1 describes the scale of observation in units of $h^{-1}$ Mpc. At large scales, one can convert the linear size $L$ to the equivalent angular scale $\theta$ in the microwave anisotropy measurements by the relation $\theta(L) \cong 1^{\circ}\left(L / 100 h^{-1} \mathrm{Mpc}\right)$. Similarly, one can associate with a scale $L$ the mass $M_{\text {smooth }}(L)=1.17 \Omega h^{2} \times 10^{12} M_{\odot}(L / M p c)^{3}$. The first column also gives $\theta(L)$ and $M_{\text {smooth }}(L)$ wherever relevant. The second and third columns list the key probes and the constraints arising from them. There are several points which need to be highlighted in this table.

If we assume that the power spectrum at large scales is $P=A k$, then COBE-DMR observations give $\sigma(R) \cong\left(24 \pm 4 h^{-1} \mathrm{Mpc} / R\right)^{2}$ at these scales (Padmanabhan \& Narasimha 1992). A straight extrapolation of this COBEDMR result to $R=50 h^{-1} \mathrm{Mpc}$ gives $\sigma_{\mathrm{COBE}}\left(50 h^{-1} \mathrm{Mpc}\right) \simeq 0.2$. On the other hand, galaxy surveys and large scale streaming motions also give useful information (Rowan-Robinson et al. 1990; Efstathiou et al. 1990; Saunders et al. 1991) around $r=50 h^{-1} \mathrm{Mpc}$. For example, the angular correlation of galaxies, measured by the APM survey is $\omega(\theta) \simeq(1-5) \times 10^{-3}$ at $\theta \simeq 14^{\circ}$. This corresponds to $\sigma_{\mathrm{G}}\left(50 h^{-1} \mathrm{Mpc}\right) \cong 0.2$. Similarly the analysis of velocity data shows (Bertschinger \& Dekel 1989; Dekel et al. 1990; Bertschinger et al. 1990) that $v\left(40 h^{-1} \mathrm{Mpc}\right) \simeq(388 \pm 67) \mathrm{kms}^{-1}$ and $v\left(60 h^{-1} \mathrm{Mpc}\right) \simeq(327 \pm 82) \mathrm{kms}^{-1}$. From these values it is possible to deduce that $\sigma_{\mathrm{LSV}}\left(50 h^{-1} \mathrm{Mpc}\right) \simeq 0.2$.

The consistency of the results, $\sigma_{\mathrm{COBE}}\left(50 h^{-1} \mathrm{Mpc}\right) \simeq 0.2$ and $\sigma_{\mathrm{LSV}}\left(50 h^{-1}\right.$ $\mathrm{Mpc}) \simeq 0.2$, is not surprising since both probe the gravitational potential of dark matter. However, the result of galaxy surveys - based on visible matter also gives $\sigma_{\mathrm{APM}}\left(50 h^{-1} \mathrm{Mpc}\right) \simeq 0.2$. This suggests observations are consistent with the conclusion that biasing is unimportant at $R \simeq 50 h^{-1} \mathrm{Mpc}$.

At small scales, the galaxy - galaxy correlation function $\xi_{\mathrm{gg}} \simeq\left[r / 5.4 h^{-1}\right.$ $\mathrm{Mpc}]^{-1.8}$ is fairly well determined. Direct observations suggest that $\sigma_{\mathrm{gal}}\left(8 h^{-1}\right.$ $\mathrm{Mpc}) \simeq 1$ but the $\sigma_{\mathrm{DM}}$ and $\sigma_{\mathrm{gal}}$ at these scales can be quite different because of biasing. In fact, one can get a direct estimate of $\sigma_{\mathrm{DM}}\left(8 h^{-1} \mathrm{Mpc}\right)$ from the abundance of rich clusters because of the following reason: the scale $R=8 h^{-1}$ Mpc contains a mass of $1.2 \times 10^{15} \Omega h_{50}^{-1} M_{\odot}$. When this scale becomes nonlinear, it will reach an overdensity of about $\delta \simeq 178$, or - equivalently - it will contract 
Table 1: OBSERVATIONAL CONSTRAINTS

\begin{tabular}{|c|c|c|}
\hline $\begin{array}{l}\text { Scale } \\
R\left(h^{-1} M p c\right) \\
\theta_{\text {CMBR }} \\
M\left(\Omega h_{30}^{-1} M_{\odot}\right) \\
\end{array}$ & Probe & $\begin{array}{l}\text { Constraint } \\
\sigma=\left\langle(\delta M / M)^{2}\right\rangle^{1 / 2}\end{array}$ \\
\hline $\begin{array}{l}R=\left(80-3 \times 10^{3}\right) \\
\theta=0.8^{\circ}-30^{\circ}\end{array}$ & $\begin{array}{l}\text { (i) COBE - DMR } \\
\text { (ii) Ground, balloon } \\
\text { observations of }(\Delta T / T)\end{array}$ & $\begin{array}{l}\text { (i) } \sigma_{D M}\left(10^{3}\right) \simeq 5 \times 10^{-4} \\
\text { (ii) } \sigma_{D M}\left(10^{2}\right) \leq 5 \times 10^{-2}\end{array}$ \\
\hline $\begin{array}{l}R=40-80 \\
\theta=0.4^{\circ}-0.8^{\circ}\end{array}$ & $\begin{array}{l}\text { (i) Galaxy surveys; e.g } \\
\text { (ii) Large scale streaming } \\
v(40)=(388 \pm 67) \mathrm{kms}^{-1} \\
v(60)=(327 \pm 82) \mathrm{kms}^{-1}\end{array}$ & $\begin{array}{l}\text { (i) } \sigma_{\mathrm{gal}}(R) ; \sigma_{\mathrm{gal}}(50) \simeq 0.2 \\
\text { (ii) } \sigma_{\mathrm{DM}}(50) \simeq 0.2\end{array}$ \\
\hline $\begin{array}{l}R=(8-40) \\
\theta=\left(4.8^{\prime}-24^{\prime}\right) \\
M=\left(10^{15}-10^{17}\right)\end{array}$ & $\begin{array}{l}\text { (i) Galaxy surveys } \\
\text { (ii) Abundance of Abell clusters }\end{array}$ & $\begin{aligned} \text { (i) } \xi_{\mathrm{gg}}=\left(r / 5 h^{-1} M p c\right)^{-1.8} & \\
\sigma_{\mathrm{gal}}(8) & \simeq 1 \\
\text { (ii) } \sigma_{\mathrm{DM}}(8) & \simeq(0.5-0.7)\end{aligned}$ \\
\hline $\begin{array}{l}R=(0.05-8) \\
M=\left(10^{8}-10^{15}\right)\end{array}$ & $\begin{array}{l}\text { (i) Abundance of qusars } \\
\text { (ii) Abundance of DLAS } \\
\text { (iii) Gunn-Peterson effect }\end{array}$ & $\begin{array}{l}\text { (i) } \sigma(0.5) \geq 3 \\
\text { (ii) } \sigma(0.5) \geq(3-4)\end{array}$ \\
\hline
\end{tabular}

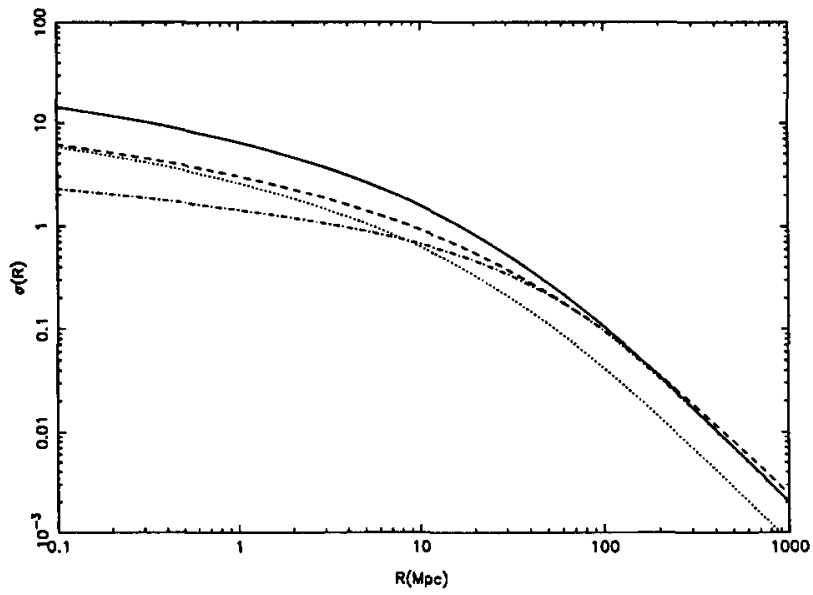

Fig.1 The linearly extrapolated density contrast, filtered by a sphere of radius $\mathrm{R}$, in four different models. All models are for $\Omega_{t}=1$, and normalised by COBE. Solid line: CDM model; Dashed line $\Lambda+$ CDM model with $\Omega_{v}=$ $0.8, \Omega_{\mathrm{CDM}}=0.2, \mathrm{~h}=0.8$; Dash-dot line : $\mathrm{C}+\mathrm{HDM}$ model with $\Omega_{\mathrm{CDM}}=$ $0.7, \Omega_{\mathrm{HDM}}=0.3 ;$ Dotted line : CDM model with $b=2.5$ 
to a radius of $R_{f} \simeq\left(8 h^{-1} \mathrm{Mpc}\right) /(178)^{1 / 3} \simeq 1.5 h^{-1} \mathrm{Mpc}$. A mass of $10^{15} M_{\odot}$ in a radius of $1.5 \mathrm{Mpc}$ is a good representation of Abell clusters we see in the universe and hence, the observed abundance of the Abell clusters can be used to constrain the value of $\sigma\left(8 h^{-1} \mathrm{Mpc}\right)$. Several people have attempted to do this (see, for a recent attempt, White et al. 1993) ; the final results vary depending on the modelling of Abell clusters, and give $\sigma\left(8 h^{-1} \mathrm{Mpc}\right) \simeq(0.5-0.7)$. Since $\sigma_{\mathrm{gal}}\left(8 h^{-1} \mathrm{Mpc}\right) \simeq 1$, this shows that $b \simeq(1.23-2)$ at $8 h^{-1} \mathrm{Mpc}$.

It is possible to present the above argument in a more general context (Subramanian \& Padmanabhan 1994). Suppose that the contribution to critical density from collapsed structures with mass larger than $M$ is $\Omega(M)$, at a given redshift $z$. Then one can show that

$$
\Omega(M)=\operatorname{erfc}\left[\frac{\delta_{c}(1+z)}{\sqrt{2} \sigma_{0}(M)}\right]
$$

where $\delta_{c}=1.68$ and $\operatorname{erfc}(\mathrm{x})$ is the complementary error function. Given the abundance $\Omega(M)$ of any class of objects at any redshift $z$, this relation can be used to determine $\sigma_{0}(M)$. The Abell clusters (at $\left.z=0\right)$ contribute in the range $\Omega \simeq(0.001-0.02)$. Even with such a wide uncertainty, we get $\sigma_{\text {clus }} \simeq(0.5-0.7)$.

One can perform a similar analysis using the abundance of quasars and damped Lyman systems. The luminosity function of quasars is fairly well determined up to $z \approx 4$. If the astrophysical processes leading to quasar formation are known, then the luminosity function can be used to estimate the abundance of host objects at these redshifts. Though these processes are somewhat uncertain, most of the models for quasar formation suggest (Hachnelt, 1993) that we must have $\sigma\left(0.5 h^{-1} \mathrm{Mpc}\right) \gtrsim 3$. Similarly, we can convert the observed $(d N / d z)$ values for damped Lyman alpha systems into abundances of host dark matter halos by making some assumptions about these objects. We find that (Subramanian \& Padmanabhan 1994) in the redshift range of $z \simeq(1.7-3.5)$ damped Lyman alpha systems contribute a fractional density of $\Omega_{L y} \simeq(0.06-0.23)$. This would require $\sigma\left(10^{12} M_{\odot}\right) \simeq(3-4.5)$.

Finally, we should remember that while we do see absorption due to clumped neutral hydrogen, quasar spectra do not show any absorption due to smoothly distributed neutral hydrogen. Since the universe became neutral at $z \lesssim z_{D} \simeq$ $10^{3}$, and since galaxy formation could not have made all the neutral hydrogen into clumps, we expect the IGM to have been ionised sometime during $5 \lessgtr$ $z \lesssim 10^{3}$ (Gunn \& Peterson 1965). It is not clear what is the source for these ionising photons. Several possible scenarios (quasars, massive primordial stars, decaying particles etc.) have been suggested in the literature though none of these appears to be completely satisfactory (Miralda-Escudé \& Ostriker 1990). In all these scenarios, it is necessary to form structures at $z \gtrsim 5$ so that an ionising flux of about $J=10^{-21} \mathrm{ergs} \mathrm{cm}^{-2} \mathrm{~s}^{-1} \mathrm{~Hz}^{-1} \mathrm{sr}^{-1}$ can be generated at these epochs. Once again, it is difficult to convert this constraint into a firm bound on $\sigma$ though it seems that $\sigma\left(0.5 h^{-1} \mathrm{Mpc}\right) \gtrsim 3$ will be necessary. 


\section{Scorecard for the Models}

The simplest models one can construct will contain a single component of dark matter, either cold or hot. Such models are ruled out by the observations. The HDM models, normalised to the COBE result will have maximum power of $\triangle_{m} \cong 0.42 h^{-2}(m / 30 e V)^{2}$ at $k=k_{m}=0.11 \mathrm{Mpc}^{-1}(m / 30 e V)$. In such a case, structures could have started forming only around $\left(1+z_{c}\right) \cong\left(\triangle_{m} / 1.68\right) \cong$ $h_{50}^{-2}(m / 30 e V)^{2}$ or at $z_{c} \cong 0$. We cannot explain a host of high- $z$ phenomena with these models. The pure CDM models face a different difficulty. These models, normalised to $\mathrm{COBE}$, predict $\sigma_{8} \simeq 1$, which is too high compared to the bounds from cluster abundance. When nonlinear effects are taken into account, one obtains $\xi_{\mathrm{gg}} \propto r^{-2.2}$ for $h=0.5$ which is too steep compared to the observed value of $\xi_{\mathrm{gg}} \propto r^{-1.8}$. In other words, CDM models have the wrong shape for $\xi(r)$ to account for the observations.

The comparison of the CDM spectrum with observations suggests that we need more power at large scales and less power at small scales. This is precisely what happens in models with both hot and cold dark matter or in models with non-zero cosmological constant. These models have been extensively studied during the last year, and they fare well as far as large and intermediate scale observations are concerned. However, they have considerably less power at small scales compared to CDM models. As a result, they do face some difficulty (Subramanian \& Padmanabhan 1994) in explaining the existence of high redshift objects like quasars and damped Lyman alpha systems. For example a model with $30 \% \mathrm{HDM}$ and $70 \% \mathrm{CDM}$ will have $\sigma_{0.5} \simeq 1.5$; to explain the abundance of damped Lyman alpha systems one needs $\sigma_{0.5} \geq 3.0$. Similar difficulties exist in models with a cosmological constant.

The comparison of models show that it is not easy to accommodate all the observations even by invoking two components to the energy density. (These models also suffer from serious problems of fine-tuning). The root cause of the difficulty is the following: the abundance of clusters requires $\sigma_{L}\left(8 h^{-1} \mathrm{Mpc}\right)$ $\simeq 0.6$ while the abundance of damped lyman alpha systems require $\sigma_{L}\left(1 h^{-1}\right.$ $\mathrm{Mpc}) \geq 3.0$. To achieve this, the mean slope of $\sigma(R)$ in the range $(1-8) h^{-1}$ Mpc should be about -0.8 . Nonlinear evolution will steepen this slope to a value much higher than the observed value of -0.9 . On the other hand, if we use models which reproduce $\sigma_{N L} \propto r^{-0.9}$, then $\sigma_{L} \propto r^{-k}$ with $k \ll 0.8$. Hence, $\sigma_{L}\left(1 h^{-1} \mathrm{Mpc}\right) \ll 3$ if $\sigma_{L}\left(8 h^{-1} \mathrm{Mpc}\right) \simeq 0.6$.

\section{Conclusions and Future Outlook}

The discussion of various models in the last section brings out three points: (1) "nice" models (e.g. pure CDM model) do not work; (2) even the "not-so-nice" models (which require fine tuning of a larger number of free parameters) are either ruled out or severely constrained; (3) the exclusion or survival of many of these models depend crucially on the estimate of errors and uncertainties in the observations.

Given these factors, it is possible to spot some of the key future observations which will be of relevance in determining the shape of the spectrum at different scales. 
(a) To begin with, the microwave anisotropy measurements are likely to improve significantly in the next few years. These observations will hopefully converge to a definite value for the primordial index $n$ (which has changed from $1.1 \pm 0.6$ to $1.59_{-0.55}^{+0.49}$ recently !), thereby allowing us to determine the shape of the spectrum at very large scales.

(b) At intermediate scales, galaxy surveys still hold the key to our understanding. What is probably required is an accurate determination of $\xi_{\mathrm{gg}}(r)$ at the scales $r \simeq(10-100) h^{-1} \mathrm{Mpc}$. At present, the attempts to obtain $\xi_{\mathrm{gg}}(r)$ from APM $\omega_{\mathrm{gg}}(\theta)$ leads to fairly large uncertainties (a factor of 5 - 10) at $r \geq 40 h^{-1} \mathrm{Mpc}$. To determine the shape of $\xi(r)$ in a useful manner we need an accuracy of better than a factor 2 or so at $r \simeq 60 h^{-1} \mathrm{Mpc}$. In this range we also have direct probes of dark matter distribution both from CMBR $1^{\circ}$ measurements and large scale streaming motions. In principle, this should allow us to make direct comparison of dark matter and baryonic distributions at these scales; in practice, it is only limited by observational and systematic errors. Surveys which go deeper than $100 h^{-1} \mathrm{Mpc}$ will, of course, be useful but only if the accuracy is reasonable.

(c) At small scales, quasars, damped Lyman alpha systems and radio galaxies provide a direct window on the high-z universe; as we have already seen most of the models have problems in explaining their abundances.

(d) If we could measure the quasar-quasar correlation function $\xi_{\mathrm{qq}}(r ; z)$ at different redshifts, we will have a direct observational probe on the evolution of correlations in the universe. At present, we may be at the threshold of getting sufficient statistical accuracy on such a project and it is well worth thinking about. Similarly, a systematic all-sky search for lensed quasars could improve our bounds on the cosmological constant.

(e) Gravitational lensing also plays a crucial role in determining the nature of dark matter via the EROS/MACHO experiments. As the statistics of events improve we should be able to put effective constraints on the amount of nonbaryonic dark matter.

\section{References}

Bagla J. S. \& Padmanabhan T., 1993, IUCAA preprint 22/93, in Proc. of VI IAU Asian Pacific Reg. Mtg, in press

Bertschinger E. \& Dekel A., 1989, ApJ, 336, L5

Bertschinger E., Dekel A., Faber S. M., Dressler A. \& Berstein D., ApJ, 1990, 364,370

Dekel A., Bertschinger E. \& Faber S. M., 1990, ApJ, 364, 349

Efstathiou G., Kaiser N., Saunders W., Lawrence A., Rowan-Robinson M., Ellis R. S. \& Frenk C. S., 1990, MNRAS, 247, 10p

Gunn J. E. \& Peterson B. A., 1965 ApJ, 142, 1633

Hachnelt M. G., 1993, IoA preprint 
Hamilton A. J. S., Kumar P., Lu E. \& Mathews A.,1991, ApJ, 374, L1

Miralda-Escudé J. \& Ostriker J. P., 1990, ApJ, 350, 1

Padmanabhan T. \& Narasimha D., 1992 MNRAS259, 41P

Rowan-Robinson M., et al., 1990, MNRAS, 247, 1

Saunders W. et al., 1991, Nature, 349, 32

Schuster J., Gaier T., Gunderson J, Meinhold P., Koch T., Seiffert M., Wueusche C. A. \& Lubin P., 1993 ApJ412, L47

Smoot G. F. et al., 1992, ApJ, 396, L1

Subramanian K. \& Padmanabhan T., 1994 IUCAA preprint No.5/94

White S. D. M., Efstathiou G. \& Frenk C. S., 1993, MNRAS, 262, 1023 